\title{
INVERSE ANALYSIS APPLIED TO AN ILLUMINATION DESIGN
}

\author{
P. S. Schneider ${ }^{\mathbf{a}}$ \\ and F. H. R. França ${ }^{b}$ \\ Universidade Federal do Rio Grande do Sul \\ Departamento de Engenharia Mecânica \\ Rua Sarmento Leite 425 \\ 90050-170 - Porto Alegre \\ Rio Grande do Sul, Brasil \\ pss@mecanica.ufrgs.bra \\ frfranca@mecanica.ufrgs.br

\begin{abstract}
This work investigates the application of the inverse analysis to an illumination design of a three-dimensional rectangular enclosure. The problem consists of finding the luminous fluxes on the light source elements, located on the top of the enclosure, that satisfies a prescribed uniform luminous flux on the design surface, located on the bottom surface. The solution assumes that all the surfaces emit and reflect diffusely, and that the hemispheric spectral emissivities are wavelength independent in the visible region of the spectrum. The inverse analysis is described by a system of linear equations that is expected to be ill-conditioned since it involves the solution of a Fredholm integral equation of the first kind. To tackle the ill-conditioned system of equations, the TSVD (Truncated Singular Value Decomposition) regularization method is applied. In addition to presenting a methodology to solve for the luminous exchanges in a enclosure, starting from a thermal radiation energy balance, this work considers two design cases: one in which the light source elements cover the entire top surface; and one in which a reduced number of light sources are considered, a more practical solution. In both cases, the proposed inverse design is capable of providing a solution that satisfies the prescribed luminous flux on the design surface within average and maximum errors less than $1.0 \%$ and $5.0 \%$, respectively.
\end{abstract}

Keywords: Inverse analysis, illumination design, luminous flux, radiation exchanges.

\section{NOMENCLATURE}

A

view factor matrix of view factors

vector of terms which are dependent on $\mathbf{x}$ conversion factor constant (683 lumens/W)

luminous power

height of the enclosure, $m$

length of the enclosure, $m$

order of the system

number of light source elements

dimensionless luminous flux

thermal radiation flux, $\mathrm{W} / \mathrm{m}^{2}$

average fluid temperature, $\mathrm{K}$

elements of the matrix $\mathbf{U}$

orthogonal matrix to $\mathbf{V}$

photopic luminous efficacy of the human eye

orthogonal matrix to $\mathbf{U}$

diagonal matrix of singular values $w_{j}$

elements of the matrix $\mathbf{W}$

width of the enclosure, $m$

coordinate direction

vector with unknown outgoing luminous fluxes on the light sources

coordinate direction

coordinate direction

\section{Greek symbols}

$\varepsilon \quad$ emissivity

$\lambda$ wavelength, $\mu \mathrm{m}$

$\gamma \quad$ relative error

$\Delta \quad$ finite-size

\begin{tabular}{ll}
\multicolumn{2}{l}{ Subscripts } \\
avg & average \\
$b$ & blackbody \\
$d$ & design surface \\
$j$ & surface element \\
$k$ & surface element \\
$l$ & lamp \\
$m$ & maximum \\
$o$ & outgoing \\
$r$ & emission minus absorption \\
$w$ & wall
\end{tabular}

\section{Superscripts}

$l \quad$ luminous flux, lumens $/ \mathbf{m}^{2}$

$w \quad$ thermal radiation flux, $\mathrm{W} / \mathrm{m}^{2}$

$T \quad$ transposed 


\section{INTRODUCTION}

The first methods for the analysis and design of artificial lighting of environments were established in the beginning of the $20^{\text {th }}$ century. Since in this early age, it was already known that the luminous flux on a given working area was not only dependent on the power of the light sources, but also on the absorbing and reflecting effect of the remaining surfaces of the enclosure. Later advances provided methods for calculation of light radiation exchanges as well as for the characterization of the light sources behavior.

In addition, many studies have been carried out to provide recommending lighting for the many possible applications, from human and industrial activities to plants growth (Boast, 1953; Mark, 2000). In general, not only the intensity of light (luminous flux intensity) is specified, but also it is also required uniformity of the lighting. The major goal of the illumination designer is to determine the position and power of the light sources so that a uniform luminous flux, at a specified value, is achieved on the working area.

The first works to deal with the illumination design were presented by Harrison and Anderson (1916 and 1920), who proposed an experimental procedure, the now called lumen method, in which the luminous flux on a working plane was determined from a combination of a series of proposed assembling of punctual and continuous light sources. In the forties, Moon (1941) and Moon and Spencer (1946a, 1946b) proposed the interreflection method for the design of threedimensional rectangular enclosures having any aspect ratio and being formed by diffuse surfaces. The method presented the advantage of allowing the calculation of the brightness of a surface, accounting for the reflection of light. Due to the complexities of the required calculations, the method required the use of tables. A solid contribution to the illumination analysis arose from the advances in the thermal radiation field in the second half of the $20^{\text {th }}$ century. This relies on the fact that light from incandescent lamps originates from the thermal radiation in the visible region of the spectrum. Therefore, the analytical and computational methods developed for the solution of thermal radiation problems in enclosures can be readily extended for the illumination. The sole difference is that the thermal radiation flux must be converted into the luminous flux, accounting for the photopic luminous efficacy of the human eye.

This work presents the application of the inverse analysis to the illumination design of an enclosure in which a uniform luminous flux, at a specified value, is imposed on a working surface of the enclosure, the design surface. As will be shown in this article, such surfaces are constrained by two boundary conditions: the specified luminous flux and the luminous emissive power, which is zero for surfaces that are not at very high temperatures. In the inverse design, the luminous power required on the light sources are found directly from the two conditions imposed on the design surfaces, without involving the trial-and-error solution of the conventional techniques. Although the inverse analysis allows some surfaces to have two prescribed boundary conditions, while other are left unconstrained, for problems that involve radiative exchanges, this type of formulation is described by a Fredholm integral equation of the first kind, known to result in an ill-posed problem, which can be solved only by means of regularization methods (Hansen, 1998). A comprehensive review of inverse design involving radiative exchanges in enclosures is presented in França et al. (2002).

This paper considers an inverse illumination design of a three-dimensional rectangular enclosure. The objective is to find the luminous fluxes on the light sources located on the top surfaces that satisfy the specified uniform luminous flux on the design surface, located on the bottom of the enclosure. All the surfaces that form the enclosure are assumed diffuse and having spectral hemispherical emissivities that are wavelength independent in the visible region of the spectrum. The work considers first a thermal energy balance in the visible light region, which is then converted to luminous quantities. A zonal type formulation is applied for the discretization of the radiation exchanges. The resulting system of equations is illconditioned due to the inverse approach that is involved. The ill-conditioned nature of the system is treated by means of the truncated singular value decomposition (TSVD). Results are presented for two cases: where the light sources cover the entire top surface; and where a reduced amount of light sources are considered, a more practical design proposition.

\section{PHYSICAL AND MATHEMATICAL MODELING}

\section{Luminous Flux and Thermal Radiation}

Incandescent lamps are a common source of light. Their main component is a resistance device that reaches very high temperatures (typically around $2900 \mathrm{~K}$ ) under the passage of electric current. At such temperatures, a considerable amount of thermal radiation is dissipated in the visible region of the wavelength spectrum, $0.4 \mu \mathrm{m} \leq \lambda \leq$ $0.7 \mu \mathrm{m}$. The luminous flux, in units of lumens $/ \mathrm{m}^{2}$ or lux, can be related to the thermal radiation flux, in units of $\mathrm{W} / \mathrm{m}^{2}$, by means of the following relation:

$$
q^{(l)}=C V_{\lambda} q^{(w)}
$$

where $q^{(l)}$ and $q^{(w)}$ correspond respectively to the luminous flux (lumens $/ \mathrm{m}^{2}$ ) and to the thermal radiation flux $\left(\mathrm{W} / \mathrm{m}^{2}\right)$ for a specific wavelength $\lambda, C$ is a conversion factor constant, equal to 683 lumens $/ \mathrm{W}$, and $V_{\lambda}$ is the so called photopic spectral luminous efficacy of the human eye, which takes into account the human eye sensitivity to the thermal radiation comprehended in the visible region of the spectrum. As shown 
in Fig. 1, the spectral luminous efficacy peaks with a value of 1.0 for a thermal radiation in the wavelength of $0.555 \mu \mathrm{m}$, and then decay monotonically to zero as the lower and upper limits of the visible region, $0.4 \mu \mathrm{m}$ and $0.7 \mu \mathrm{m}$, are approached.

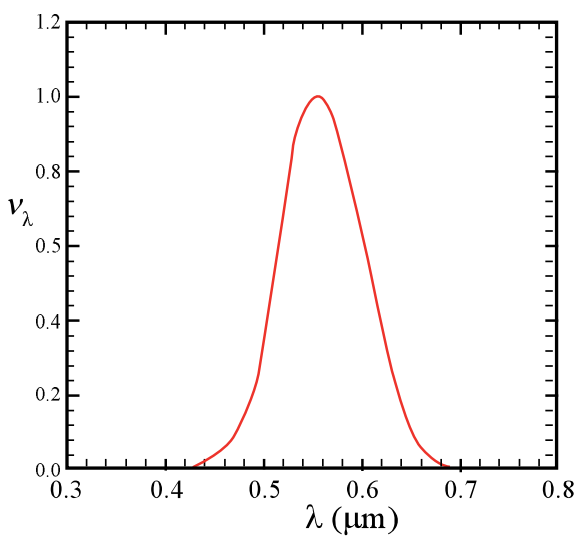

Figure 1. Photopic luminous efficacy of the human eye.

In general, a source of light is composed by radiation covering the entire range of the visible region. In such a case, Eq. (1) must be applied to each infinitesimal amount of the spectral energy and then be integrated in the visible spectrum. Such procedure will be demonstrated in the next section.

\section{RADIATION EXCHANGES IN AN ENCLOSURE}

Procedures for the evaluation of radiation exchanges in an enclosure are well established in the modern literature (Siegel and Howell, 2002). In this work, this will be accomplished by first subdividing the enclosure into sufficiently small elements, so that all thermal quantities can be assumed uniform. Next, the energy balance will be applied to each element $j$ of the enclosure. Depending whether the temperature or the net radiative heat flux are known, the energy balance can take the following forms, respectively:

or

$$
q_{\lambda, o, j}^{(w)}=\varepsilon_{\lambda, j} e_{\lambda, b, j}^{(w)}+\left(1-\varepsilon_{\lambda, j}\right) \sum_{k} F_{j-k} q_{\lambda, o, k}^{(w)}
$$

$$
q_{\lambda, o, j}^{(w)}=q_{\lambda, r, j}^{(w)}+\sum_{k} F_{j-k} q_{\lambda, o, k}^{(w)}
$$

where $q_{\lambda, o}^{(w)}$ is the spectral outgoing radiative heat flux, in $\mathrm{W} /$ $\left(\mathrm{m}^{2} \mu \mathrm{m}\right)$, which takes into account both emission and reflection; $q_{\lambda, r}^{(w)}$ is the spectral net radiative heat flux, in $\mathrm{W} /\left(\mathrm{m}^{2} \mu \mathrm{m}\right)$, which takes into account emission minus absorption; $e_{\lambda, b}^{(w)}$ is the spectral blackbody emissive power, in $\mathrm{W} /\left(\mathrm{m}^{2} \mu \mathrm{m}\right)$, which is solely dependent on the temperature of the surface element; $\varepsilon_{\lambda}$ is the spectral emissivity; and $F_{j-k}$ is the view factor between surface elements $j$ and $k$. In the above equations the superscript $(w)$ was maintained in all energy terms to indicate thermal energy quantities. In a next step, such terms will be converted into luminous quantities. To accomplish this, it should be first noted that the thermal energy flux $\left(\mathrm{W} / \mathrm{m}^{2}\right)$ in the wavelength $\lambda$ centered in the interval $d \lambda$ can be related to the spectral energy flux by:

$$
d q^{(w)}=q_{\lambda}^{(w)} d \lambda
$$

The corresponding luminous flux (lumens) in the wavelength $\lambda$ centered in the interval $d \lambda$ follows from Eq. (1):

$$
d q^{(l)}=C V_{\lambda} q_{\lambda}^{(w)} d \lambda
$$

Finally the total luminous flux is obtained through the integration of the above equation in the entire visible region:

$$
q^{(l)}=\int_{\lambda=0.4 \mu \mathrm{m}}^{0.7 \mu \mathrm{m}} C V{ }_{\lambda} q_{\lambda}^{(w)} d \lambda
$$

Applying steps (4) to (6) to all the spectral thermal energy fluxes of Eqs. (2) and (3), one obtains the energy balance in terms of the luminous fluxes:

or

$$
q_{o, j}^{(l)}=\varepsilon_{j} e_{b, j}^{(l)}+\left(1-\varepsilon_{j}\right) \sum_{k} F_{j-k} q_{o, k}^{(l)}
$$

$$
q_{o, j}^{(l)}=q_{r, j}^{(l)}+\sum_{k} F_{j-k} q_{o, k}^{(l)}
$$

where $q_{o}^{(l)}$ is the outgoing luminous flux, in lumens $/ \mathrm{m}^{2}$ or lux, which takes into account both emission and reflection; $q_{r}^{(l)}$ is the net luminous flux, in lumens $/ \mathrm{m}^{2}$, which takes into account emission minus absorption; $e_{b}^{(l)}$ is the blackbody luminous power, in lumens $/ \mathrm{m}^{2}$, which is solely dependent on the temperature. To obtain Eqs. (7) and (8), it was assumed that the spectral emissivity $\varepsilon_{j}$ was independent of the wavelength in the visible region of the spectrum.

\section{PROBLEM DEFINITION}

Figure 2 presents a schematic view of a three-dimensional enclosure, which is formed by surfaces that are diffuse and have spectral hemispherical emissivities that are wavelength independent in the visible region of the spectrum. The design surface, where a luminous flux is to be specified, is located on the bottom of the enclosure; the incandescent lamps, the light sources, are located on the top surface. The remaining of the enclosure is formed by walls that do not emit but reflect incident light. The length, width and height of the enclosure are designated by $L, W$ and $H$, respectively. 
As depicted in Fig. 3, the enclosure is divided into finite-size square elements, $\Delta x=\Delta y=\Delta z$, in which the luminous energy balance will be applied. For designation of elements in the design surface, lamps and wall, indices $j d, j l$ and $j w$ will be used throughout this analysis.

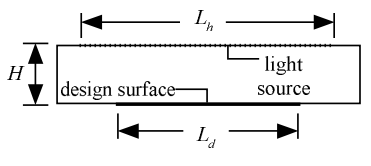

Lateral View

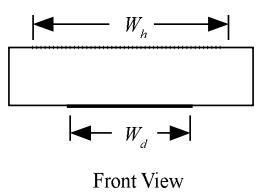

Figure 2. Three-dimensional rectangular enclosure.

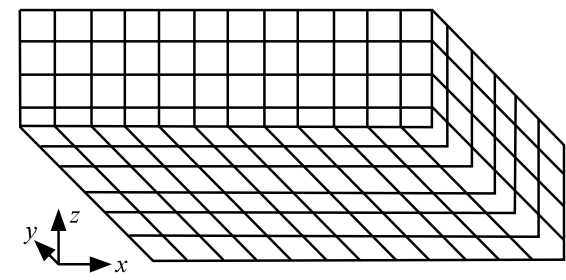

Figure 3. Division of the bottom and two side surfaces of the enclosure into finite size elements.

In this analysis, it is considered that an uniform luminous flux (in lumens $/ \mathrm{m}^{2}$ or lux), designated by $q_{\text {specified }}^{(l)}$, is specified on the design surface. The problem consists of finding the luminous power on each light source element located on the top surface so that this requirement is achieved. The luminous exchanges in the enclosure are governed by Eqs. (7) and (8).

In illumination designs, the net luminous flux $q_{r}^{(l)}$ is imposed on the design surface. In addition, the temperatures of the elements on the design surface are well below the temperatures for each there is thermal radiation in the visible region; so, the blackbody luminous power is also known, that is, $e_{b}^{(l)}=0$. Therefore, two conditions are imposed on the design surface elements. For the elements on the wall, one single conditions is known, $e_{b}^{(l)}=0$, since they do not emit light. On the other hand, no condition is known for the light source elements. In fact, the conditions on the light sources are to be found from the two specifications $\left(q^{(l)}=q_{\text {specified }}^{(l)}\right.$ and $e_{b}^{(l)}=0$ ) on the design surface.

In conventional solution techniques, one and only one condition (either the net luminous flux, $q_{r, j}^{(l)}$, or the blackbody luminous power, $e_{b, j}^{(l)}$ ) is imposed on each surface element $j$. This leads to a system of equations on the unknown outgoing luminous flux of each surface $j, q_{o, j}^{(l)}$, formed by Eqs. (7) and (8). This system is in general well-behaved and can be solved by any standard matrix inversion technique, such as Gaussian elimination, or by iterative techniques. Once the system is solved for the outgoing luminous fluxes, Eqs. (7) and (8) can be again applied to find the unknown condition (the net luminous flux or the blackbody luminous power) of each surface element. Since the knowledge of one condition is required on each surface element, the conventional technique can tackle the illumination design only through a trial-and-error solution, in which the net luminous flux is guessed on each light source, and the solution is run to verify if the imposed luminous flux on the design surface was attained. Due to the nature of the problem, one should expect to make many guesses to obtain an approximate solution.

The inverse design is an alternative technique to tackle the above problem without relying on a trial-and-error solution, although the mathematics of the problem require special solution methods, as will be discussed in Section 4 . To demonstrate the inverse design approach, one needs first to combine Eqs. (7) and (8) for a design surface element $j d$ to relate the outgoing luminous flux to the blackbody luminous power and the net luminous flux; that is:

$$
q_{o, j d}^{(l)}=e_{b, j d}^{(l)}-\frac{1-\varepsilon_{j d}}{\varepsilon_{j d}} q_{r, j d}^{(l)}
$$

Since $e_{b, j d}^{(l)}=0$, the outgoing luminous flux can be found directly from the known net luminous flux. Writing the above equation in dimensionless form, one obtains:

$$
Q_{o, j d}=-\frac{1-\varepsilon_{j d}}{\varepsilon_{j d}} Q_{r, j d}
$$

where the dimensionless luminous flux is given by $Q=q^{(l)} / q_{\text {specified }}^{(l)}$. Note from this definition that $Q_{r, j d}=-1.0$. The negative signal arises from the fact that the energy balance was based on the convention that luminous flux out of the surface is positive.

Next, Eq. (8) is applied to each design surface element $j d$, and then rearranged to provide a system of equations on the dimensionless outgoing luminous flux on the light source elements $Q_{o, j l}$ :

$$
\sum_{j l} F_{j d-j l} Q_{o, j l}=\left(Q_{o, j d}-Q_{r, j d}\right)-\sum_{j w} F_{j d-j w} Q_{o, j w}
$$

Note that in the above equation, the first two terms on the right-hand side are known. The outgoing luminous flux on the wall elements, $Q_{o, j w}$, are unknown, so an additional relation can be imposed by applying Eq. (7) to these elements and imposing the condition that $e_{b, j w}^{(l)}=0$; that is:

$$
\begin{gathered}
Q_{o, j w}=\left(1-\varepsilon_{j w}\right)\left(\sum_{j d} F_{j w-j d} Q_{o, j d}+\right. \\
\left.\sum_{j l} F_{j w-j l} Q_{o, j l}+\sum_{j w-j w^{\prime}} F_{j w-j w^{\prime}} Q_{o, j w^{\prime}}\right)
\end{gathered}
$$


The last term arises from the fact that, as seen in Fig. 1, two wall element $j w$ and $j w$ ' can "see" each other, in which case the view factor is not zero. Finally, the set of equations become complete setting Eq. (8) for each light source element $j l$ :

$$
Q_{r, j l}=Q_{o, j l}-\sum_{j d} F_{j l-j d} Q_{o, j d}-\sum_{j w} F_{j l-j w} Q_{o, j w}
$$

\section{SOLUTION PROCEDURE}

Based on the procedure proposed in França et al. (2002), the following approach is adopted. The outgoing luminous flux on the elements located on the walls are initially neglected in Eq. (11). Therefore, the unknowns in Eq. (11) are only the outgoing luminous fluxes on the light source elements, $Q_{o, j l}$. Once Eq. (11) is written for each of the $M$ elements that form the design surface, a system with $M$ equations will be formed. The unknowns are the outgoing radiative heat fluxes on the $N$ light source elements. Therefore, one aspect of the inverse design arises: the number of equations and the number of unknowns are not necessarily the same, unless $M=N$. In addition, since the problem corresponds to a discrete form of a integral equation of the first-kind, one should expect the system of equations to be ill-conditioned. In most cases, such systems cannot be solved by standard matricial solvers, but rather should be treated by regularization methods, like the Tikhonov method, the conjugate gradient, the truncated singular value decomposition (TSVD), the modified TSVD, among others. The chosen method of solution, TSVD, is discussed in the next section, which is a well explored method to deal with ill-conditoned non-square system of equations.

Although a system of equations can be assembled in such a way to include the equations for the elements on the walls, an aspect of interest in the aforementioned procedure is that the elements in the design surface, where two conditions are imposed, are directly linked to the light source elements, which are left unconstrained. In other words, the ill-posed part of the problem is separated from the remaining of the problem, allowing an isolated application of the appropriate methods of solution of ill-posed system of equations.

The solution is achieved by means of the following procedure. First, the outgoing luminous flux on each design surface element $j d$ is computed by means of Eq. (10). As discussed above, the outgoing luminous flux on each wall element, $Q_{o, j w}$, is initially set equal to zero. Then, the system of equations formed by Eqs. (11) is solved for the outgoing radiative heat fluxes on the heaters, $Q_{o, j l}$. Next, Eq. (12) is applied to each wall element $j w$ to form a system of equations on the outgoing luminous flux on the wall elements, $Q_{o, j w}$ . Once the system is solved, the newly computed $Q_{o, j w}$ are inserted into the system of equations formed by Eqs. (11), and the procedure is repeated until convergence is achieved. Finally, with the converged values of the outgoing luminous fluxes, Eq. (13) is applied to solve for the net luminous flux on each light source element.

\section{REGULARIZATION OF THE SYSTEM OF EQUA- TIONS}

The procedure discussed above involves the solution of a system of linear equations on the outgoing luminous fluxes on the light sources, as formed by Eqs. (11), which can be represented by:

$$
\mathrm{A} \cdot \mathrm{x}=\mathrm{b}(\mathrm{x})
$$

where matrix $\mathbf{A}$ is formed by the view factors between the design surface and the light source elements, $F_{j d-j l}$; vector $\mathbf{x}$ represents the unknown outgoing luminous fluxes on the light sources, $Q_{o, j l}$; and vector $\mathbf{b}$ contains the terms on the righthand side of Eq. (11), which are dependent on $\mathbf{x}$.

The system formed by Eqs. (11) is the discretized form of a set of equations that includes Fredholm integral equations of the first kind, and so it is expected to present the characteristics of ill-posed problems. In general, the components of the exact solution vector $\mathbf{x}$ present steep oscillations between very large positive and negative numbers, and small perturbations cause a much amplified change in the solution. One solving an ill-posed problems should not aim at an exact solution, but rather to impose additional constraints to reduce the size (norm) of $\mathbf{x}$, and achieve a smooth solution. However, the greater the smoothness imposed on the solution is, the greater will be the residual. This is the basic idea behind any regularization method for the solution of ill-posed problems.

Among the regularization procedures, the truncated singular values decomposition (TSVD) is employed here. First, matrix $\mathbf{A}$ is decomposed into three matrices:

$$
\mathrm{A}=\mathrm{U} \cdot \mathrm{W} \cdot \mathrm{V}^{\mathrm{T}}
$$

where $\mathbf{U}$ and $\mathbf{V}$ are orthogonal matrices, and $\mathbf{W}$ is a diagonal matrix formed by the singular values $w_{j}$. As a consequence, the solution vector $\mathbf{x}$ can be computed by:

$$
\mathbf{x}=\sum_{j=1}^{N}\left(\frac{b_{k} \cdot u_{k j}}{w_{j}}\right) \mathbf{v}_{j}
$$

where $N$ stands for the number of unknowns (in this case, the number of light source elements). 
As will be demonstrated, in ill-posed problems, the singular values $w_{j}$ decay continuously to very small values. Since they appear in the denominator of Eq. (16), the components of $\mathbf{x}$ can present very large absolute numbers. However, the smaller the singular value $w_{j}$ is, the closer the corresponding vector $\mathbf{v}_{j}$ is to the null-space of $\mathbf{A}$. In other words, the terms related to the smaller singular values can be eliminated from Eq. (16) without introducing a large error into the solution. This is the main idea of the TSVD: only the terms related to the $p$-th largest singular values are kept on Eq. (16), instead of all $N$ terms. The solution is the vector $\mathbf{x}$ with the smallest norm subjected to minimum deviation $\mid \mathrm{A} \cdot \mathrm{x}-\mathrm{b}$.

Another aspect of the inverse solution concerns the number of unknowns and the number of equations. The number of equations equals the number of unknowns only when the number of light source elements and the number of design surface elements are the same. In general, this is not always verified, as in the example cases to be shown later in this work. An important feature of the TSVD method is that it can also be applied to the situation where the numbers of unknowns and equations are not the same, as will be shown in the results section.

\section{VERIFICATION OF THE SOLUTION}

Due to the need for regularization of the system of equations, an exact solution is not expected. The following procedure is used for the verification of the solution. Once the net luminous fluxes on the light source elements are obtained, a forward problem is solved where the net luminous fluxes on the light source elements are known, and only the condition $e_{b}^{(l)}=0$ is imposed on the design surface (as well as on the wall elements). The net luminous flux on each element $j d$ of the design surface is then calculated, and compared to the specified heat flux by:

$$
\gamma_{j d}=\left|\frac{Q_{r, \text { specified }}-Q_{r, j d}}{Q_{r, \text { specified }}}\right|
$$

where $Q_{r, \text { specified }}$ is the specified luminous flux, equal to -1.0 , and $Q_{r, j d}$ is the luminous flux resulting from the conditions on the light sources that were obtained in the inverse solution. Once $\gamma_{j d}$ is calculated for each element $j d$ in the design surface, the arithmetic average and the maximum errors $\gamma_{\text {avg }}$ and $\gamma_{\max }$ can be readily found.

\section{RESULTS AND DISCUSSION}

The case considered in this work consists of a threedimensional enclosure as shown in the schematic of Fig. 1. The aspect ratio of the enclosure base is $W / L=0.8$; the dimensionless height is $H / L=0.2$. The selection of the other dimensions of the enclosure will require a few considerations. First, the design surface ought not to cover the entire extension of the base, since the portions close to the corners would be mainly affected by the reflections from the sidewalls, not from the luminous radiation from the light source elements on the top surface. Therefore, the design surface dimensions are taken as $L_{d} / L=0.8$ and $W_{d} L=0.6$. The amount and positions of the light sources will be proposed later on.

The boundary conditions follow from the discussion in Section 3: for the design surface elements, the luminous emissive power is zero and the dimensionless net luminous flux is $Q_{r, j d}=-1.0$; for the wall elements, the luminous emissive power is also zero; the light source elements are left unconstrained. The hemispherical emissivities in the visible light region of the design surface, of the light sources and of the walls are $\varepsilon_{d}=0.9, \varepsilon_{l}=0.9$ and $\varepsilon_{w}=0.5$, respectively. The problem is at this point completely defined but for the location of the light source elements, which will be discussed next.

\section{CASE 1: LIGHT SOURCES COVERING THE ENTIRE TOP SURFACE}

To illustrate the inverse design, it is first considered that the light source elements cover the entire top surface. Figure 4a shows the locations of both the light source elements (circular dots) and the design surface elements (shaded area).

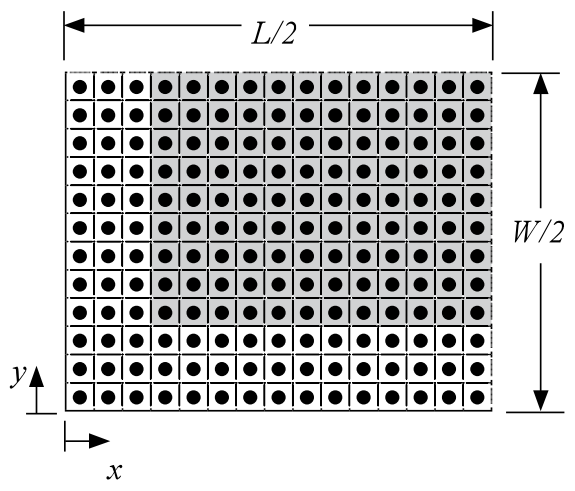

Figure 4. Locations of the design surface (shaded area) and light source elements (circular dots) in one quarter of the bottom and top surfaces for case 1: light sources covering the entire top surface. Dashed lines indicate symmetry.

Due to the problem symmetry, indicated by the dashed lines, only a quarter of the domain needs to be solved: $0 \leq x / L \leq$ $0.5,0 \leq y / L \leq 0.4)$. One consequence of having the light sources covering the entire top surface is that the numbers of design surface and light source elements ( $M$ and $N$, respectively) are not the same. Selecting a grid size of $\Delta x / L=1 / 30$, one finds $M=108$ and $N=180$. Thus, the system of equations formed by Eq. (11) will be composed by $M=108$ equations and $N=180$ unknowns. 
The procedure presented in the former section is then applied to find the required net luminous flux on the light source elements. Figure 5 shows the singular values of matrix $\mathbf{A}$, under the label Case 1. As typical of inverse design problems, the singular values $w_{j}$ decay steadily to values that are as small as $10^{-9}$. It should be noted that in the figure only the non-zero singular values are shown; for $j>M=108$, all the singular values are zero. This indicates that this mathematical problem presents infinite exact solutions. To select the exact solution with the smallest norm, one needs only to apply the TSVD method to retain only the $M$ first terms of the series of Eq. (16). Even taking such a measure, it was observed that the components of vector $\mathbf{x}$ had very large absolute values, and alternating positive and negative signals, due the small singular values that arise in the denominator of the series terms. Since the components of vector $\mathbf{x}$ correspond to the outgoing luminous fluxes on the light sources, a positive number, this exact solution has no physical, not to say practical, meaning. However, the TSVD can be further explored to keep only the terms of the series corresponding to the $p(<M)$ largest singular values. In this case, the obtained regularized solution will be no longer exact, but can present more acceptable behavior.

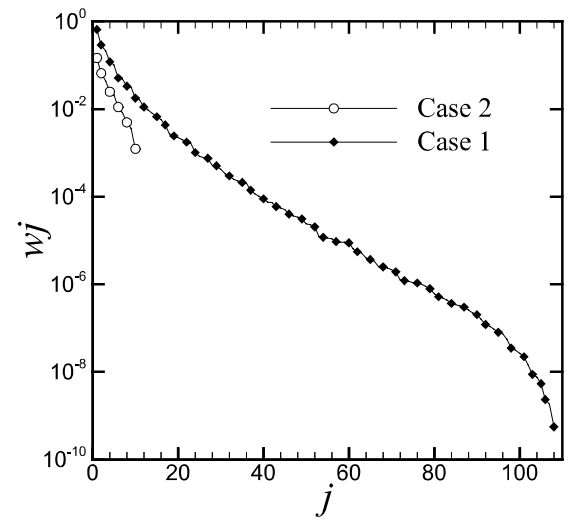

Figure 5. Singular values of matrix $\mathbf{A}$ : cases 1 and 2.

Figure 6 presents examples of regularized solutions for different regularization parameters $p=14,12,10$ and 8 . Solutions for $p \geq 15$ presented negative values for the outgoing luminous lux on some of the light sources, and they were not considered. Solutions for $p=13,11$ and 9 were also obtained, but since they do not bring additional features to those shown in Fig. 6, they are not presented here. As can be seen in all cases, all the solutions present an oscillatory behavior that are in fact a reminiscent of the steep oscillations of the smallest-norm exact solution (that is, for $p=M=108$ ). In general, the smaller the value $p$ is, the smoother the solution will be, although at the expense of presenting a larger error. (a)

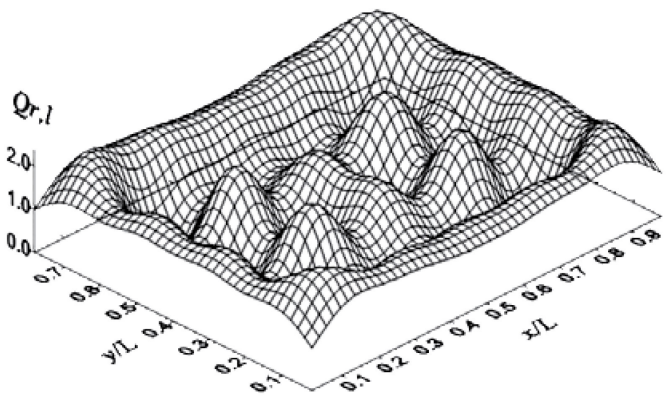

(b)

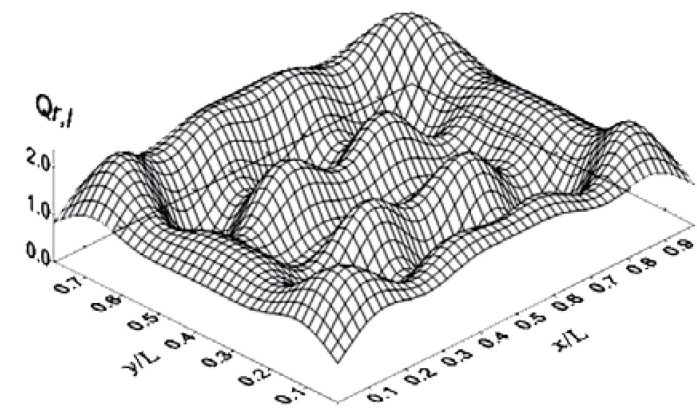

(c)

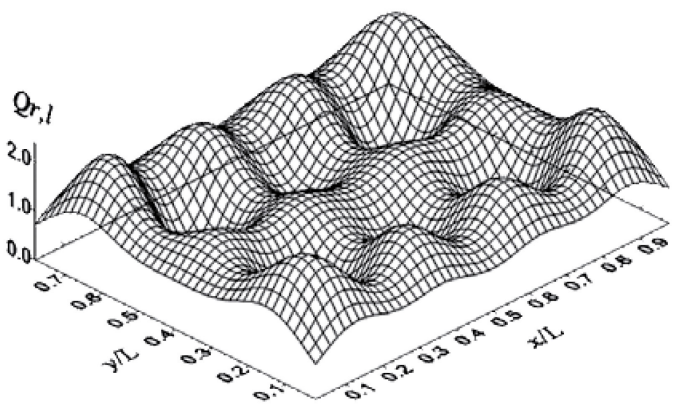

(d)

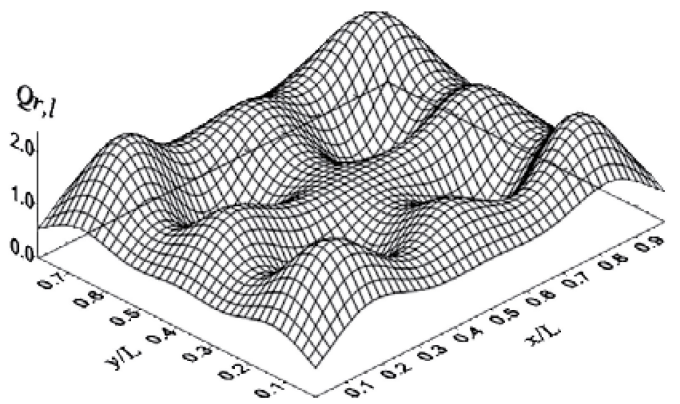

Figure 6. Required net luminous flux distribution on the top surface for different regularization parameters $p$ : (a) $p=14$; (b) $p=12 ;$ (c) $p=10 ;$ (d) $p=8$. Case 1 . 
This can be verified in Tab. (1), which presents the maximum and average errors for different values of $p$, as defined in Section 4.2, as well the minimum singular value retained in the series of Eq. (16), $w_{j \text { min }}$. Although the errors for $p=14$ and 12 are the smallest ones, the corresponding solutions for the net luminous flux on the light sources showed a few negative values, which is not a practical solution. Such negative net luminous flux on the light sources were not present for the solutions corresponding to $p=10$ and 8 . Since, between these two, the solution for $p=10$ presented the smallest error, this could be the selected one among the four solutions in Fig. 6.

Table 1. Minimum singular value and inverse solution errors for different regularization parameters $p$.

\begin{tabular}{|c|c|c|c|}
\hline$p$ & $w_{j, \min }$ & $\gamma_{\mathrm{avg}}(\%)$ & $\gamma_{\max }(\%)$ \\
\hline 14 & $7.9274 \times 10^{-3}$ & 0.1932 & 1.2714 \\
\hline 12 & $1.1154 \times 10^{-2}$ & 0.3873 & 1.9053 \\
\hline 10 & $1.7734 \times 10^{-2}$ & 0.6244 & 2.7894 \\
\hline 8 & $3.3388 \times 10^{-2}$ & 1.1689 & 4.8468 \\
\hline
\end{tabular}

\section{CASE 2: REDUCED NUMBER OF LIGHT SOURCES}

The solutions obtained and discussed in the previous section illustrate the typical fact that the inverse design technique can lead to a number of approximate solutions, where the final selection can take into account the accuracy and practicality of the solutions. One aspect of the light source configuration of Fig. 4 is that a very large number of independent light sources are being required (that is, $4 \times 180=$ 720 elements for the entire enclosure), which is not practical. The designer will probably be much more interested on having a smaller amount of heat sources. For that matter, the previously selected solution (for $p=10$ ) might provide a hint towards this goal. Indeed, the fact that only ten combinations were enough to provide a solution with acceptable error is an indication that a solution with only ten light sources might be enough to satisfy the problem within an acceptable solution. Figure 7 illustrates a proposed design where ten light sources are distributed on the one quarter of the top surface. The locations of the light sources were proposed to approximately coincide with the points of maximum luminous flux of the solution in Fig.6c.

Since the number of elements on the design remains the same, $M=108$, and the number of light sources is $N=10$, the problem is overspecified; that is, the number of equations $M$ is larger than that of unknowns. It is well known that such a problem can only be solved with some approximation. Figure 5 presents the singular values of matrix $\mathbf{A}$ for this case, under the label of Case 2. As seen, the ten singular values decay to a value in the order of $10^{-3}$. Keeping all the singular values in the series of Eq. (16) provides the smallest norm vector $\mathbf{x}$ that satisfies the smallest norm $|\mathrm{A} \cdot \mathrm{x}-\mathrm{b}|$.

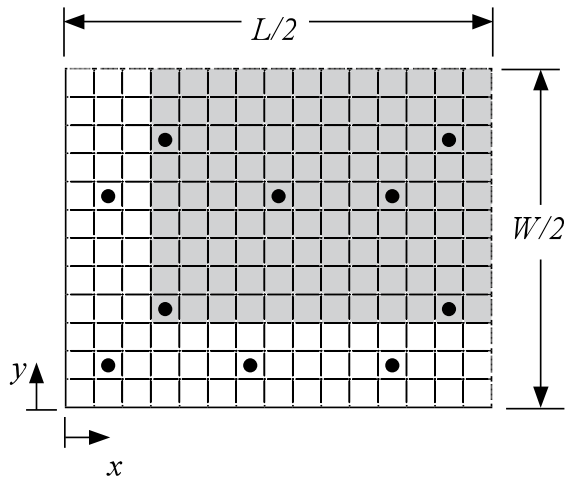

Figure 7. Locations of the design surface (shaded area) and light source elements (circular dots) in one quarter of the bottom and top surfaces for case 2 : reduced number of light sources. Dashed lines indicate symmetry.

The solution is shown in Tab. (2), which indicates the net luminous flux on each element shown in Fig. 7. In the table, the location of the light sources are indicated by indices $i$ and $j$ to designate the position in $x$ and $y$ directions, respectively.

Table 2. Required dimensionless net luminous on the light source elements for case 2 .

\begin{tabular}{|c|c|c|c|c|c|c|c|}
\hline$J l$ & $i$ & $j$ & $Q_{r, j}$ & $j l$ & $i$ & $j$ & $Q_{r . j}$ \\
\hline 1 & 2 & 2 & 17.4009 & 6 & 8 & 8 & 20.8525 \\
\hline 2 & 2 & 8 & 11.7953 & 7 & 10 & 2 & 15.7124 \\
\hline 3 & 4 & 4 & 31.1068 & 8 & 10 & 8 & 31.2191 \\
\hline 4 & 4 & 10 & 36.6861 & 9 & 14 & 4 & 24.5422 \\
\hline 5 & 8 & 2 & 35.6220 & 10 & 14 & 10 & 9.1672 \\
\hline
\end{tabular}

The resulting luminous flux on the design surface is shown in Fig. 8. As seen the solution is within the interval $-0.95<Q_{r, j d}<-1.05$; the maximum and average error of the solution, as discussed in Section 4.2, are $4.0322 \%$ and $0.9867 \%$. If an error less $5.0 \%$ is required, then the proposed solution is acceptable. Note that other solutions can be tried for other locations of the light sources, so an even better solution can be found. 


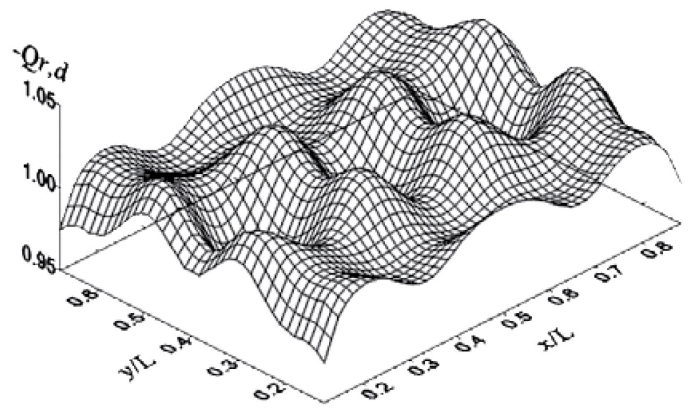

Figure 8. Dimensionless net luminous flux on the design surface for the solution in case 2 .

All the above solutions involved a discretization of the spatial domain such that $\Delta x / L=1 / 30$, which proved to be sufficiently small to guarantee grid independence. As a final remark, it should be pointed that, for greater generality, all the results were presented in dimensionless form, but conversion dimensional terms should be straightforward. For instance, the luminous power (in lumens) on each light source element of Tab. (2) can be determined as $Q_{r, j l} \cdot q_{\text {specified }}^{(l)} \cdot(\Delta x)^{2}$.

\section{CONCLUSIONS}

This work considered a inverse design problem in which the net luminous fluxes on the light source elements were determined to satisfy the specified uniform luminous flux on the design surface. The numerical discretization of the problem led to an ill-conditioned system of equations as it is usual of an inverse design approach. Such systems cannot be solved by conventional matrix solvers, requiring regularization methods, such as the truncated singular valued decomposition (TSVD). The TSVD eliminates the linear combinations related to the smallest singular values of the system, keeping only the $p$ largest ones. The regularization of the system inevitably introduces some error, but makes it possible to obtain useful and accurate solutions.

The proposed procedure set a system of equations relating the design surface elements directly to the light source elements, calculating the remaining terms, that is, the outgoing luminous fluxes on the wall elements, from the conditions of the previous iterative step. Because the ill-conditioned part of the problem was isolated, it was necessary to apply regularization only to a reduced system of equations.

Two design cases were studied. In case 1 , it was considered that the light source elements covered the entire top surface. As a consequence, the number of unknowns (the net luminous fluxes on the light sources) was larger than the number of equations (the luminous energy balance applied to each design surface element). The TSVD method was applied to find the solution for different regularization parameters, resulting in a few solution that were evaluated according to physical requirements (outgoing luminous fluxes to be positive), practical requirements (net luminous fluxes to be positive on the light sources), and the error of the solution. The selected solutions were obtained while keeping only ten terms in the series that form the solution vector. In case 2 , it was proposed an inverse design in which only ten light source elements were set on the top surface (case 2). Like in the selected solution in case 1 , the solution vector was obtained from a series having only ten terms. Despite the problem becoming over specified, it was possible to obtain a solution being much more practical than having light sources covering the entire top surface, and still presenting an average and a maximum error less than $1.0 \%$ and $5.0 \%$, respectively. It would be very difficult to arrive at such a solution by a trial-and-error procedure of conventional techniques.

In possible next steps, the proposed inverse design analysis can be applied to consider the effect of external illumination, to include surfaces that present both specular and diffuse reflection characteristics, to take into account the directional and/or wavelength dependency of the surface emissivities, and to consider the problem of finding the optimum location of the light sources.

\section{ACKNOWLEDGMENTS}

The authors thank CAPES (Brazil) for the support under the program CAPES/UT-AUSTIN, No. 06/02.

\section{REFERENCES}

Boast W.B, 1953, Illumination Engineering, McGraw-Hill, New York.

França, F., Howell, J., Ezekoye, O., and Morales, J. C., 2002, Inverse Design of Thermal Systems, in:Advances in Heat Transfer, J. P. Hartnett and J. P. Irvine, eds., 36, Elsevier, pp. 1-110.

Hansen, P. C., 1990, Truncated SVD Solutions to Discrete Ill-Posed Problems with Ill-Determined Numerical Rank, SIAM J. Sci. Statist. Comput., Vol. 11, pp. 503-518.

Harrison, W. and Anderson, E. A., 1916, Illumination Efficiencies as Determinated in an Experimental Room, Trans. Illum. Eng. Soc., Vol. 11, pp. 67-91.

Harris on W. and Anderson E. A., 1920, Coefficients of Utilization, Trans. Illum. Eng. Soc., Vol. 15, pp. 97-123.

Mark, S., 2000, The IESNA Lighting Handbook, Illuminating Engineering Society of North America, New York.

Moon P., 1941, Interreflections in Rooms, J. Optical Soc. Am., Vol. 31, pp. 374-382.

Moon, P., and Spencer, D. E., 1946a, Light Distribution in Rooms, J. Franklin Inst., Vol. 242, pp. 111-141.

Moon, P., and Spencer, D. E., 1946b, Light Design by the Interreflection Method, J. Franklin Inst., Vol. 242, pp. 465-501.

Siegel, R., and Howell, J. R., 2002, Thermal Radiation Heat Transfer, 4th Ed., Hemisphere Publishing Corporation, Washington.

Received: September 06, 2006

Revised: October 06, 2006

Accepted: November 06, 2006 